MODELING, IDENTIFICATION AND CONTROL, 1993, VOL. 14, NO. 3, 121-131

doi:10.4173/mic.1993.3.1

\title{
A comparison of strategies for the control of a polypropene reactor $\dagger$
}

\author{
BERNT LIE‡ and JENS G. BALCHEN§
}

Keywords: Process control, control structures, polymer control.

Several controller structures for a polypropene reactor system are evaluated, and it is shown that the present controller structure with retuned controller parameters yields very good performance with regards to set point tracking, and suppression of disturbances and model uncertainty. Multivariable LQG control with integral action shows even better results, and would have given excellent performance without some observed limitation in the estimator.

\section{Introduction}

The industrial polypropene CSTR reactor under investigation is at present controlled by means of several single-loop PI-controllers. Propene feed (monomer) is used to control the reactor slurry level, while the heat of reaction is removed by refluxing the necessary amount of reactor vapour through an external condensation device. The liquid level in the reflux accumulator is controlled as well. Thus, the control of three properties is studied in this paper: reactor level, reactor pressure, and accumulator liquid level. The control inputs are: monomer feed, the signal to a splitrange valve arrangement, and the efflux of condensed liquid from the reflux accumulator. The process can be described in a block diagram as depicted in Fig. 1. Further details about the system are provided in Appendix A.

The present control structure consists of 3 single loop controllers, each from output $y_{i}$ to input $u_{i}$. In this paper, the following additional structures are investigated: (improved tuning of the present control structure,) pressure control by directly

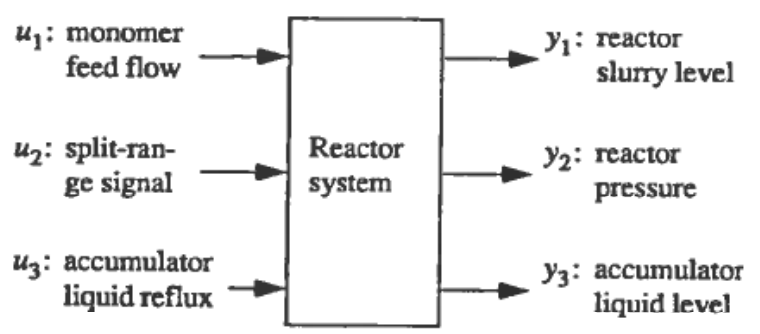

Figure 1. Block diagram of polypropene reactor.

Received 28 February 1993.

$\dagger$ Rewritten and expanded version of a paper presented at IFAC symposium DYCORD +'92, April 26-28, 1992, College Park, Maryland, USA. Reprinted with permission from Pergamon Press.

$\ddagger$ Department of Process Automation, Telemark Institute of Technology, Kjølnes, N-3914 Porsgrunn, Norway.

$\S$ Department of Engineering Cybernetics, The Norwegian Institute of Technology, N-7034 Trondheim, Norway. 
manipulating heat removal (single loop controllers from $y_{1}$ to $u_{1}$, from $y_{2}$ to $u_{3}$, and from $y_{3}$ to $u_{2}$ ), multivariable PID-control synthesized by asymptotic LQG-methods, and modified nonlinear decoupling.

The paper is organized as follows: In Section 2, the different controller structures for the polypropene reactor are discussed briefly, starting with physical reasoning over the possible single loop structures. Section 3 provides a comparison of the structures by simulation. Finally, the results are discussed and some conclusions are drawn.

\section{Control strategies}

\subsection{Single loop structures}

Control input $u_{1}$ provides mass influx, but also cools down the process: the feed is colder than the reactor. Input $u_{2}$ controls heat removal from the system by determining the fraction of reactor vapor reflux that passes through an external heat exchanger/ condenser, as well as the rate of condensation and liquid influx to the accumulator. Input $u_{3}$ controls the mass efflux from the accumulator, as well as the cooling of the reactor: accumulator liquid is colder than the reactor content.

In general, it is a good idea to control the amount of material in the system (i.e., levels) by means of the material flows into or out of the system. This is due to the fact that levels are only weakly related to the energy content of the system: liquid density and condensation/vaporization influence levels, but liquid density is not very sensitive to temperature variations, and the heat of vaporization is usually large.

Since $u_{1}$ controls the total accumulated mass in the reactor system, $u_{1}$ should be used to control the reactor level $y_{1}$. When the refluxed accumulator liquid reenters the reactor and is thus heated up to the energy level of the reactor, this heat is taken from the accumulated energy in the reactor. At the same time, the system energy content is reduced in the reflux condenser. Manipulation of reflux accumulator liquid flow should therefore give fast control of the reactor energy content by using the accumulator liquid as a cooling reservoir. Manipulation of energy removal in the reflux condenser should give a somewhat slower response, since the removed reactor energy will be smoothed through the accumulator level. In the simulation results, the present structure (controlling $y_{2}$ with $u_{2}$, and $y_{2}$ with $u_{3}$ ) is denoted present. The application of $u_{2}$ to control $y_{3}$ and $u_{3}$ to control $y_{2}$ is termed direct-energy, since $u_{3}$ (reflux liquid flow) directly controls reactor energy content.

It turns out that the tuning of the present structure can be improved. In the simulations, this controller setting is denoted pres-improve.

Other methods of selecting pairing in single loop control, are discussed (Lie (1990) and Lie and Balchen (1991)).

\subsection{Multivariable PID-controller}

A general multivariable PID-controller is depicted in Fig. 2. Derivative action is achieved by using state feedback. Feedback from estimated states leads to 'filtered' derivative action.

The feedback gains $G_{x}$ (proportional gain) and $G_{z}$ (reset gain) are found by computing LQ optimal feedback for the linearized system, and tuning the control weight by varying a scalar $\rho(\rho \rightarrow 0$ implies no control effort weight). Tuning by varying $\rho$ is introduced in order to achieve acceptable performance vs. robustness properties.

The observer gain $K$ is computed from the Kalman filter equations. A crude cstimate of process disturbance and measurement noise statistics are inferred from 


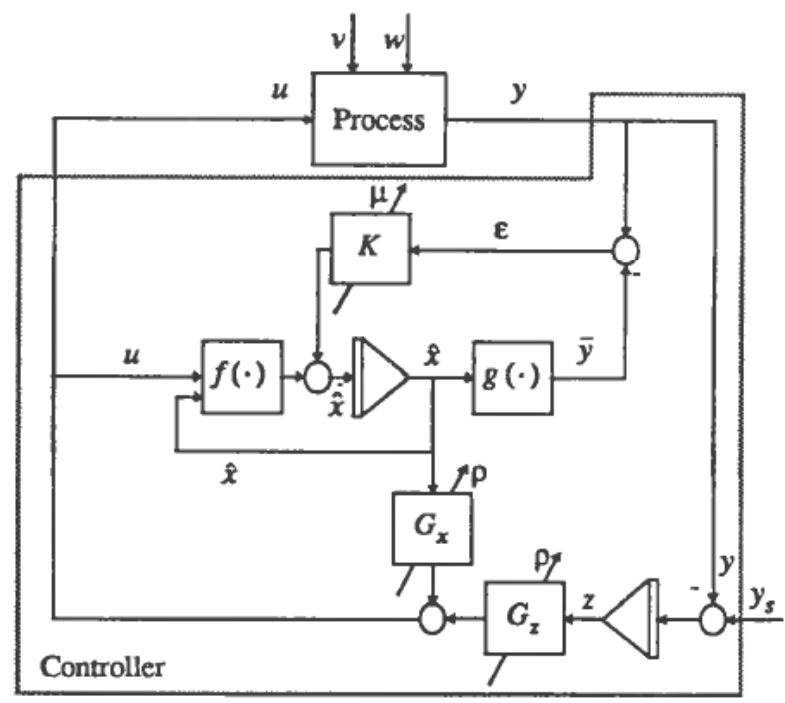

Figure 2. The structure of the multivariable PID-controller, with tuning of the Kalman filter gain and the feedback gains.

knowledge of measurement uncertainty and reasonable rate of change of states; the statistics matrices are primarily used to scale the weighting when solving the observer Riccati equation. As when solving the LQ problem, a tuning parameter $\mu$ is introduced, and varied in order to achieve acceptable observer performance ( $\mu \rightarrow 0$ implies no measurement uncertainty).

In the simulations, this strategy is denoted LQG-PID.

\subsection{Decoupling}

In nonlinear decoupling, the coupling in the system is removed by introducing a nonlinear feedback compensation, see, e.g., Balchen et al. (1988). The resulting system consists of decoupled integrators, and the system can be given the desired closed loop response. For the polypropene system, this strategy turns out to be quite robust, Lie and Balchen (1990), although the decoupling may be lost due to uncertainty. In the present investigation, state estimates are used in the decoupling, and the set point signals of the system are low pass filtered. In the simulations, this strategy is denoted decoupling.

\section{Evaluation of the strategies by simulation}

\subsection{Tests of performance}

A number of methods exist for the analytic evaluation of system robustness and performance, e.g., application of $\mathrm{H}_{\infty}$ theory, Lyapunov theory, etc. Such methods are difficult to apply to nonlinear systems/systems of high order, and simulated experiments are widely used to evaluate their performance. It is, however, impossible to undertake a complete investigation of the quality of a strategy by simulation, as this would involve an infinite number of experiments. A set of tests must therefore be chosen which reveal the strengths and weaknesses of a structure.

When comparing the different control structures, the following qualities are believed to be important: set point step response, disturbance rejection, and the 
suppression of parameter changes/model changes. In this paper, the response in reactor pressure, reactor slurry level and accumulator liquid level are displayed. The excitations are a step change in pressure set point, a step change in feed temperature (e.g., a sudden rainfall), and a step change in reaction frequency factor (e.g., poisoning of the catalyst). See Lie (1990) for further details, other excitations, and responses in other quantities.

\subsection{Pressure set point tracking}

The pressure set point is varied in order to change the rate of polymer production. Here, the pressure set point is changed suddenly from $32-33.5$. The response in reactor slurry level is shown in Fig. 3. The response in reactor pressure is shown in Fig. 4. The response in accumulator level is depicted in Fig. 5.

When using a multiple control structure (several single loop controllers), both the present structure with improved controller settings and a suggested new structure with a more direct control of the removed energy are seen to yield a good response. If large step changes are likely to occur in the pressure set point, it may be advantageous with a larger liquid volume (energy buffer) in the reflux accumulator. Overall, the simplest and cheapest way to improve the pressure response is to fine tune the pressure loop gain of the present structure. Experiments with the simulation model suggest that the pressure loop gain can be more than doubled in the present structure.

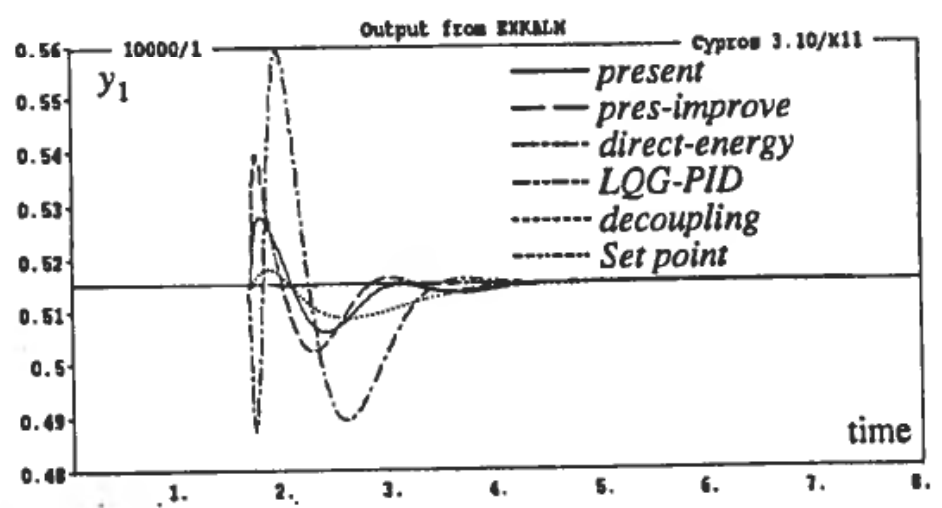

Figure 3. The response in the reactor slurry level to a step change in the reactor pressure set point.

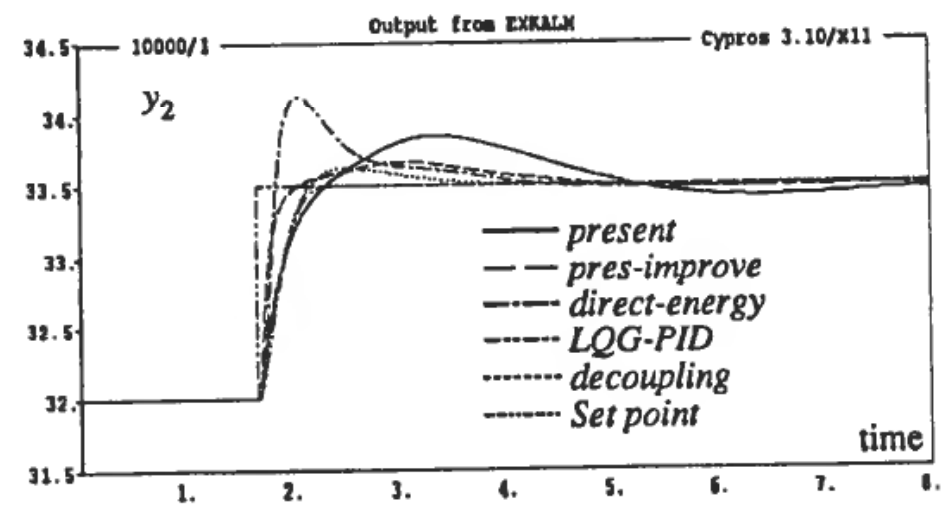

Figure 4. The response in the reactor pressure to a step change in the reactor pressure set point. 


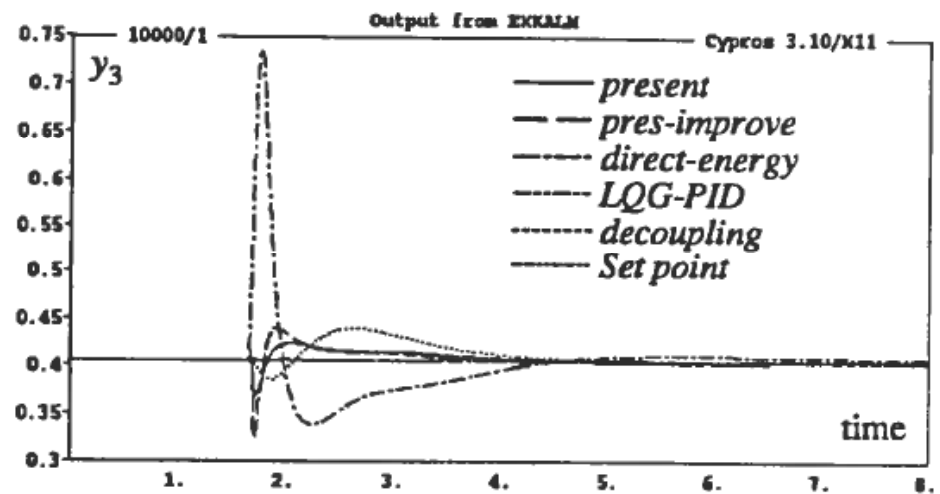

Figure 5. The response in the measured accumulator level to a step change in the reactor pressure set point.

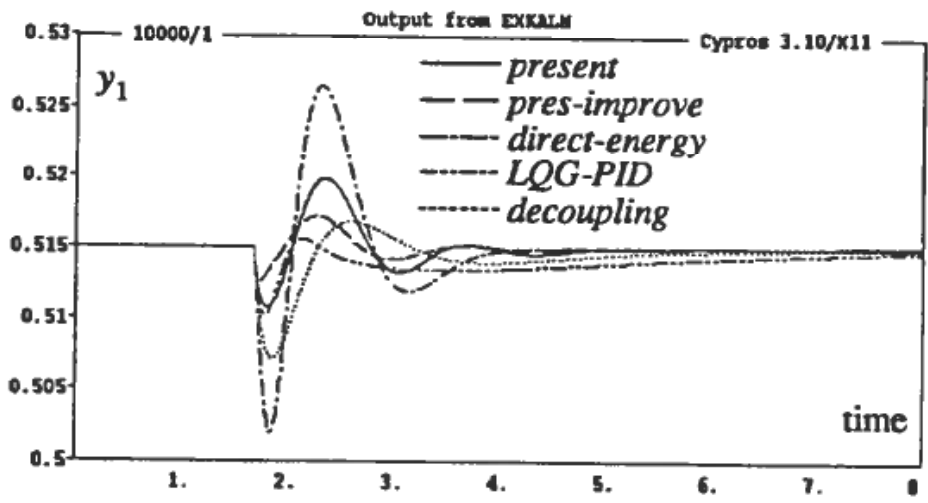

Figure 6. The response in the reactor slurry level to a step change in the feed flow temperature.

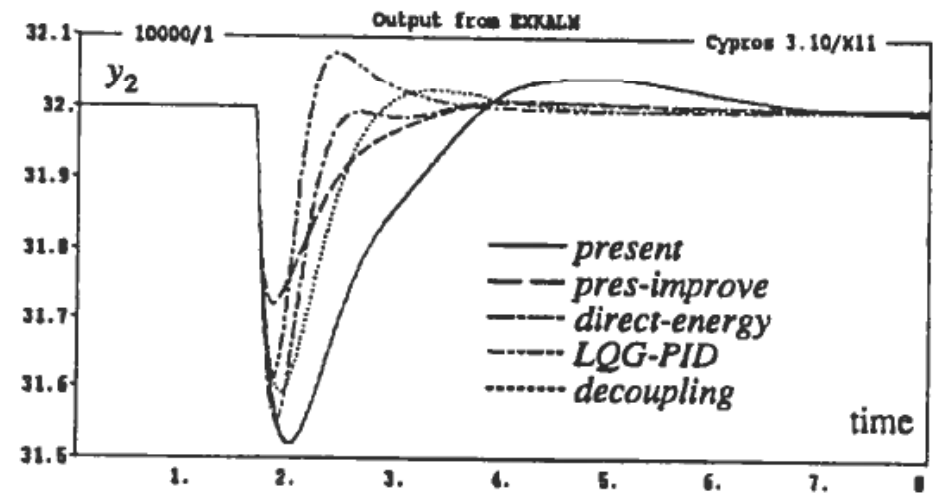

Figure 7. The response in the reactor pressure to a step change in the feed flow tempcrature. 


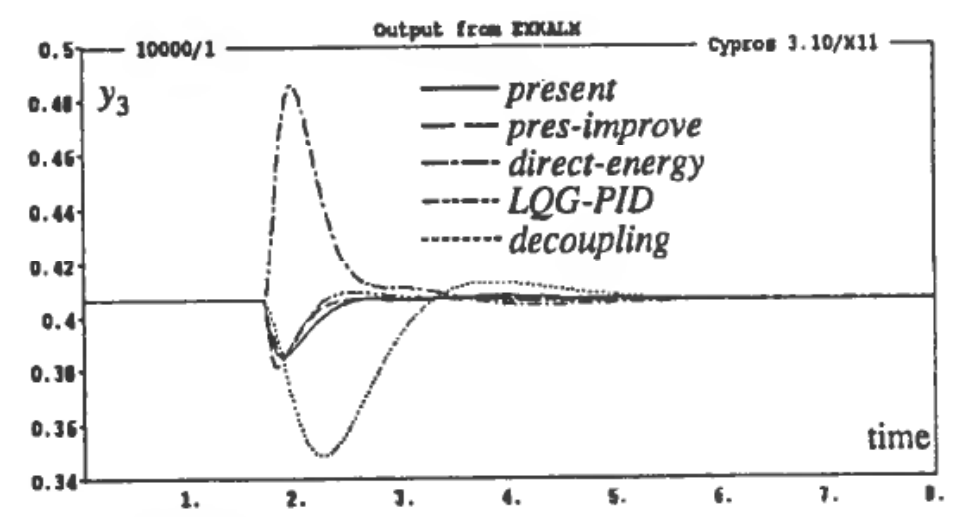

Figure 8. The response in the accumulator level to a step change in the feed flow temperature.

By iteratively varying the weight on the manipulating variables in linear quadratic control, a multivariable PID controller exhibits an unparalleled rejection of upsets in the outputs to changes in the pressure set point. At the same time, the response in the pressure is very precise and smooth. The possible disadvantage of this structure is the more complicated algorithms that should be implemented in a computer.

Application of the decoupling strategy shows the second best responses. The reason why perfect decoupling is not achieved lies in the sensitivity of this structure to small model errors. Still, the response is very good. Experiments revealed that it is vital to low-pass filter the set point changes to avoid excessive use of the control signals. This strategy is as complex to implement as that of the multivariable PID controller.

\subsection{Rejection of feed temperature change}

The feed temperature is assumed to change suddenly from $20^{\circ} \mathrm{C}$ to $0^{\circ} \mathrm{C}$. The response in reactor slurry level is given in Fig. 6. The response in reactor pressure is shown in Fig. 7. The response in accumulator level is illustrated in Fig. 8.

The present structure with a better tuning of the control parameters appears to best suppress the upsets due to a sudden change in the monomer feed temperature. On the other hand, the multivariable PID controller best rejects upsets due to a change in the catalyst feed flow (Lie 1990). The difference between the structures is not dramatically large. The suggested new multiple structure and the decoupling strategy does, however, allow for a rather large upset in the accumulator liquid level.

\subsection{Suppression of a change in reaction frequency factor}

The catalyst activity is assumed to increase (depoisoning) suddenly from $370 \mathrm{~h}^{-1}$ to $500 h^{-1}$. The resulting response in reactor slurry level is shown in Fig. 9. Figure 10 displays the response in reactor pressure. Accumulator liquid level response is given in Fig. 11.

The multiple structures appear to give the best overall rejection of changes in the process parameters. This is due to their relatively low dependence on the accuracy of the model, compared to the multivariable structures. When only the pressure output is considered, the suggested new structure of controlling the pressure by the liquid reflux flow/energy yields the best result. The upset in the accumulator liquid level is considerable, however. 


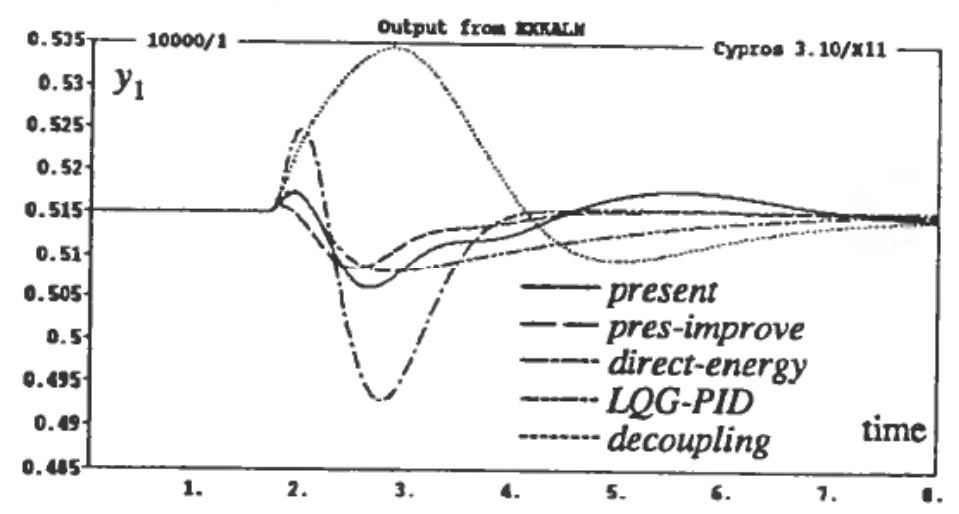

Figure 9. The response in the reactor slurry level to a step change in the reaction frequency factor.

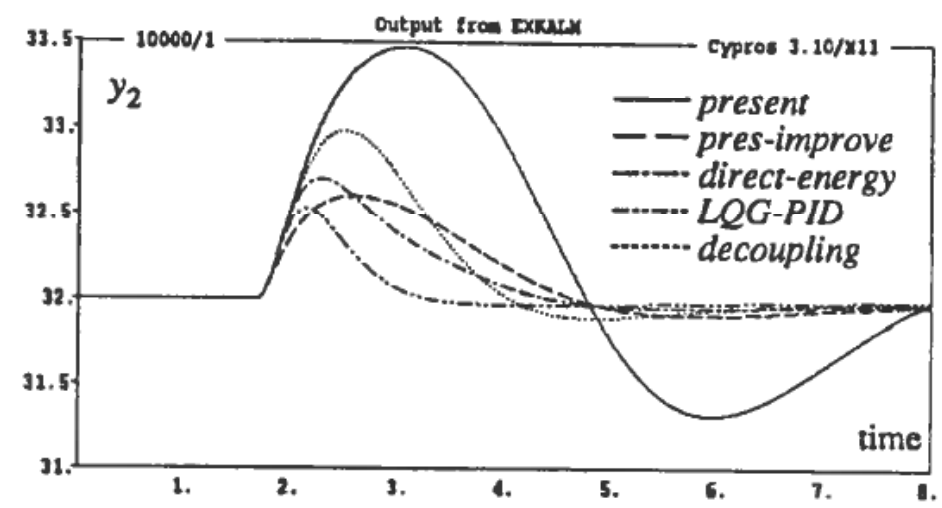

Figure 10. The response in the reactor pressure to a step change in the reaction frequency factor.

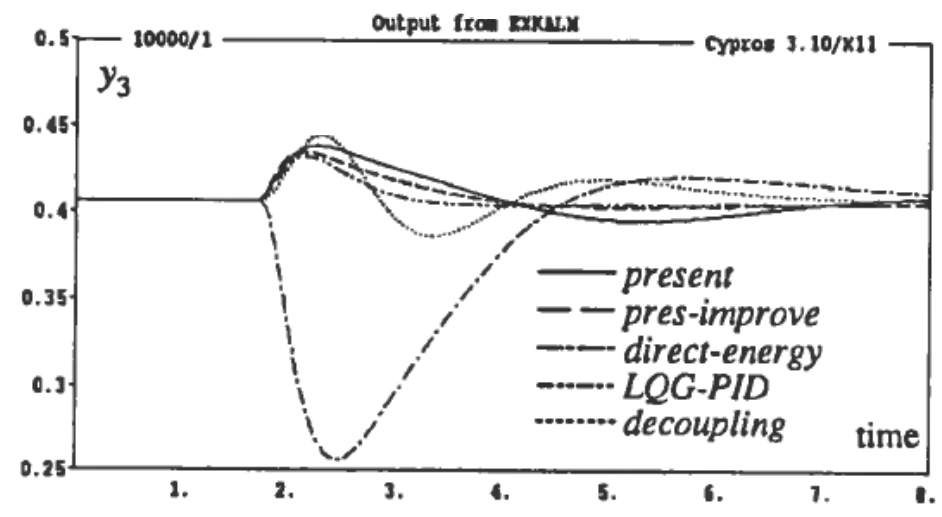

Figure 11. The response in the accumulator liquid level to a step change in the reaction frequency factor. 
The multivariable PID controller gives a good rejection of changes in the reaction frequency factor, but does not handle sudden changes in the heat transfer coefficient very well (Lie 1990). The decoupling strategy does not give a good suppression of the level upsets, but handles the pressure upsets reasonably well.

\section{Conclusions}

The simulations reported in Section 3 show that by refining the tuning of the present control structure, this strategy can be dramatically improved. The suggested direct control of removed energy also gives an improved system, but somewhat inferior to the present structure. Thus, improvements in the existing structure should be executed without delay since this can be done at negligible cost.

Overall, the linear quadratic based multivariable PID controller is the most promising control structure for governing basic properties of the polypropene reactor system. This structure gives superior set point tracking, while simultaneously ensuring quite good rejection of disturbances and process parameter changes. In the longer term, it is recommended that this structure is implemented.

The decoupling structure also provides a satisfactory performance, and one of its unique advantages in the daily operation of the process should be considered. The outer loop of the decoupling structure will essentially consist of decoupled systems, thus its behaviour may be found more predictable and be simpler to tune by the human operator.

The observed reduction in performance when using feedback from estimated states (Lie, 1990) is investigated in a forthcoming paper. Essentially, it is found that by using two additional measurements (monomer mass of reactor slurry, and catalyst mass in reactor slurry), the observed reduction in performance is partially removed.

\section{REFERENCES}

BALCHEN, J. G., LIE, B., and SOLBERG, I. (1988). Internal decoupling in non-linear process control. Modeling, Identification and Control, 9, 137-148.

FLORY, P. J. (1953). Principles of Polymer Chemistry (Cornell University Press, London).

LIE, B. (1990). Control structures for polymerization processes applied to polypropene manufacturing. Ph.D. thesis, Div. Engn. Cyb., Norwegian Institute of Technology.

LIE, B., and BALCHEN, J. G. (1990). Robustness of nonlinear decoupling in a polypropene reactor. In J. E. Rijnsdorp, J. F. MacGregor, B. D. Tyreus and T. Takamatsu (editors): Proceedings, Dycord +' '89, Maastricht, The Netherlands, August 21-23, 1989 (Pergamon Press, Oxford).

Lie, B., and Balchen, J. G. (1991). The Pairing Problem in Process Control: a Critical Survey. Paper presented at the AIChE annual meeting, Los Angeles, 1991.

Papadopoulou, S. A., and Gilles, E. D. (1986). Continuous Estimation of the Chain Length Distribution in a Polymerization Reactor Considering Time Discrete Gel-Permeation Chromatographic Measurements. In: K.-H. Reichert and W. Geiseler (editors): Polymer Reaction Engineering. Hüthig \& Wepf Verlag, Basel.

\section{Appendix A. The polypropene reactor system}

\section{A.1. The process}

Polypropene is produced in a continuous stirred tank reactor. The reaction medium consists of monomer (propene), catalyst $\left(\mathrm{TiCl}_{3}\right)$, cocatalyst (DEAC), and some impurities (ethene, ethane, propane, nitrogen, etc.). Hydrogen is added in order to terminate the growing polymers and thus control the chain length. Heat is removed by 


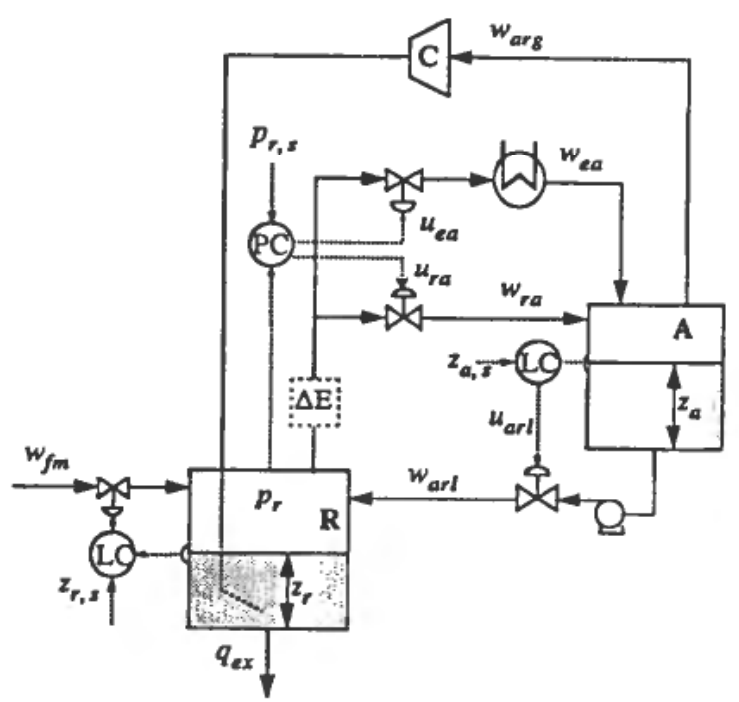

Figure 12. The main control loops of the polypropene reactor.

means of an overhead condensor in a reflux loop. Good mixing is achieved by using an impeller and by refluxing gas through the reactor slurry (reaction medium and precipitated polymer). The reactor system is sketched in Fig. 12.

The conversion in the reactor is not complete, and the unreacted propene is purified before it re-enters the reactor. The cost may be reduced by increasing the conversion, i.e., by increasing the pressure (and thereby the temperature) of the reactor.

A common assumption in polymer modeling is that both the rate of propagation and the heat of reaction are independent of the chain length (Flory 1953). Basic states like temperature and levels are therefore independent of the chain length of the polymer, see Papadopoulou and Gilles (1986).

The model of the reactor system is based upon the following assumptions:

- The system contains monomer, polymer, inert, and nonvolatile catalysts.

- The vapor phase and the liquid phase of the monomer are at equilibrium.

- There are no spatial gradients within the phases.

- The condensation in the heat exchanger can be described by an algebraic expression.

- The amount of inert in the reactor can be calculated from the difference between the observed pressure and the monomer saturation pressure at the reaction temperature.

Obviously this model will not give a perfect description of the process, since we know that there will indeed be gradients and impurities. Still it may be sufficiently accurate for the purpose of controlling the reactor.

The chosen basic states in the model are: the mass of monomer in the reactor, the mass of catalyst, the mass of polymer, the temperature of the reactor, the mass of monomer in the reflux accumulator, the temperature of the accumulator. Finally, one state describes an observed deviation in the temperature sensitivity of the reaction rate from that described in the literature.

See Lie (1990) for further details on the process model. 


\section{A.2. The linearized system}

In order to have an analytic method to select the pairing, it is advantageous that a linear(ized) model of the system is available. In state space, the model may be written as:

$$
\begin{aligned}
& \dot{x}=A x+B u+\Gamma v \\
& y=C x+w
\end{aligned}
$$

where:

- $x^{\mathrm{T}}=\left[\Delta m_{r}, \Delta m_{k}, \Delta m_{s}, \Delta T_{r}, \Delta m_{a}, \Delta T_{a}, \Delta x_{r}\right]$

- $u^{\mathrm{T}}=\left[\Delta w_{f m}, \Delta u_{s}, \Delta w_{a r l}\right]$

- $v^{\mathrm{T}}=\left[\Delta T_{f}, \Delta w_{w}, \Delta w_{f k}, \Delta w_{u}\right]$

- $y^{\mathrm{T}}=\left[\Delta z_{r}, \Delta p_{r}, \Delta z_{a}\right]$

For arbitrary variable $q, \Delta q$ denotes the deviation from its steady state value. $x$ denotes state variables, $u$ denotes control variables, $v$ denotes disturbances, $y$ denotes measurements, and $w$ denotes measurement noise.

States: $m_{r}$ is monomer mass in the reactor, $m_{k}$ is catalyst mass in the reactor, $m_{s}$ is mass of solid (polymer) in the reactor, $T_{r}$ is the reactor temperature, $m_{a}$ is the mass of monomer in the accumulator, $T_{a}$ is the accumulator temperature, and $x_{r}$ is an auxiliary state representing the resistance in the film around the polymer particles.

Control variables: $w_{f m}$ is the mass feed flow of monomer to the reactor, $u_{s}$ is the signal to the split range valves, and $w_{a r l}$ is the flow of liquid from the accumulator to the reactor.

Disturbances: $T_{f}$ is the feed temperature, $w_{w}$ is mass flow of water to the overhead condenser, $w_{f k}$ is mass feed flow of catalyst, and $w_{u}$ is the mass flow of unreacted monomer out of the reactor.

Measurements: $z_{r}$ is the level of slurry in the reactor, $p_{r}$ is the reactor pressure, and $z_{a}$ is the liquid level in the accumulator.

The matrices are:

$$
A \approx\left(\begin{array}{ccccccc}
0 & 0 & 0 & -3080 & 0 & 3170 & 1 \\
430 \times 10^{-6} & -0 \cdot 471 & 0 & 0 & 0 & 0 & 0 \\
0 \cdot 349 & 0 & -0 \cdot 471 & 0 & 0 & 0 & -1 \\
0 & 0 & 0 & -7 \cdot 50 & 0 & 8 \cdot 49 & -19 \cdot 6 \times 10^{-3} \\
0 & 0 & 0 & 3080 & 0 & -3170 & 0 \\
0 & 0 & 0 & 35 \cdot 7 & 0 & -67 \cdot 7 & 0 \\
0 & -381 & 0 & -353 & 0 & 0 & -1
\end{array}\right)
$$




$$
\begin{aligned}
& B \approx\left(\begin{array}{ccc}
1 & 0 & 1 \\
0 & 0 & 0 \\
0 & 0 & 0 \\
-1.45 \times 10^{-3} & 0 & -0.479 \times 10^{-3} \\
0 & 0 & -1 \\
0 & 2730 & 0 \\
0 & 0 & 0
\end{array}\right) \\
& \Gamma^{\mathrm{T}} \approx\left(\begin{array}{cccccc|c}
0 & 0 & 0 & 29.9 \times 10^{-6} & 0 & 0 & 0 \\
0 & 0 & 0 & 0 & 0 & -0.202 \times 10^{-3} \\
0 & 1 & 0 & 0 & 0 & 0 & 0 \\
-1 & -0.915 \times 10^{-3} & -0.740 & 0 & 0 & 0 & 0 \\
0
\end{array}\right. \\
& C^{\mathrm{T}} \approx\left(\begin{array}{ccc}
74.9 \times 10^{-6} & 0 & 0 \\
0 & 0 & 0 \\
32.9 \times 10^{-6} & 0 & 0 \\
12.3 \times 10^{-3} & 0.486 & 0 \\
0 & 0 & 539 \times 10^{-6} \\
0 & 0 & 5.75 \times 10^{-3} \\
0 & 0 & 0
\end{array}\right)
\end{aligned}
$$

For simplicity, the gain $B^{\prime \prime}$ from the computed controller signal to the valve position/flow rates has been drawn out of the system:

$$
B^{\prime \prime} \approx \operatorname{diag}\left[12 \times 10^{3}, 4.87 \times 10^{-3}, 33 \cdot 4 \times 10^{3}\right]
$$

i.e., the real input matrix $B$ is the one reported above post multiplied by $B^{\prime \prime}$. 